\title{
INFLUENCIA DE DOS COMPOSTAS EN LA GERMINACIÓN DE SEMILLAS DE ALBAHACA (Ocimum basilicum L.) Y ORÉGANO SILVESTRE (Lippia graveolens
}

\author{
Kunth)
}

\author{
Influence of two compostes in the germination of seeds of basil (Ocimum \\ basilicum 1.) and wild oregano (Lippia graveolens kunth)
}

Luis Guillermo Ríos-Villaseñor, Alicia de Luna-Vega, José Pablo Torres-Morán, Ana Karen Rodríguez-Ponce y Martha Isabel Torres-Morán*

Centro Universitario de Ciencias Biológicas y Agropecuarias (CUCBA) Camino Ramón Padilla Sánchez No. 2100 Nextipac, Zapopan, Jalisco C.P.45200.

*Autor para correspondencia: isabel.torres@academicos.udg.mx

\section{Resumen}

En la germinación de semillas, existe poca información sobre cuales procesos son directamente afectados $\mathrm{o}$ beneficiados por la composta y la calidad de la misma. En el presente trabajo se realizó una prueba del efecto del uso de composta durante el proceso de germinación y crecimiento de dos especies aromáticas: la albahaca (Ocimum basilicum L.) y el orégano silvestre (Lippia graveolens Kunth.). Las compostas usadas, fueron elaboradas a partir de estiércol de conejo y de borrego. Los resultados obtenidos probaron la influencia de las compostas en la germinación y el crecimiento de las plántulas de albahaca y orégano silvestre, éste último reportado como especie de muy difícil germinación. Se tuvieron incrementos del $20 \%$ en el porcentaje de germinación en orégano y de más del 10\% en albahaca. También se encontraron resultados satisfactorios en cuanto a peso seco de orégano y longitud de brote en las plántulas de albahaca.
Palabras clave: Sustratos, semillas de hortalizas, materia orgánica, composta de conejo, composta de borrego

\begin{abstract}
Currently, there is a lack of information regarding the positive of negative influence of compost and its quality in seed germination. This paper presents the results stemming from the utilization of compost during the stages of germination and growth of two aromatic spices: of Basil (Ocimum basilicum L.) and wild oregano seeds (Lippia graveolens Kunth.). The compost used in these tests were derived from rabbit and sheep manure. The results obtained showed the influence of composts on the germination and growth of Basil and wild oregano seedlings, the latter reported as being more difficult to germinate. Germination percentage and initial growth of seedlings were measured. Increases of $20 \%$ in the percentage of germination in oregano were found and more than $10 \%$ in basil compared with control treatment without compost. Satisfactory results were also found in dry oregano weight and sprout length in basil seedlings.
\end{abstract}


Keywords: Substrates, vegetable seeds, organic matter, sheep manure compost, rabbit manure compost.

\section{Introducción}

La materia orgánica es la fuente de energía y alimento de los microorganismos del suelo además mejora la estructura del suelo ya que lo protege de la erosión y compactación, lo hace más poroso para facilitar la aireación, retención de humedad y capacidad de intercambio catiónico. Reduce la necesidad de la utilización de fertilizantes químicos, amortigua el cambio de $\mathrm{pH}$ del suelo y además, los ácidos orgánicos del humus ayudan a disolver los minerales del suelo (De Luna y Vázquez, 2009).

El compostaje es una técnica prometedora para reducir las emisiones contaminantes de los desechos sólidos de animales. La principal ventaja del compostaje es que la mayoría de los desechos generados por las granjas pueden ser compostados sin pretratamiento antes de su uso. El compostaje no es un proceso unitario simple, es la suma total de una serie de procesos metabólicos complejos y transformaciones que se produce como resultado de la actividad de una gran población mixta de microorganismos. Los cambios químicos precisos, y las especies microbianas involucradas en ellos, varían de acuerdo a la composición de los materiales de compostaje (Solano y col., 2001).

La germinación se define como el desarrollo de las estructuras esenciales del embrión para producir una nueva plántula bajo condiciones favorables, hasta el momento de la emergencia. La terminación del reposo e iniciación de la germinación se lleva a cabo una vez que se presentan las condiciones que estimulan los procesos de crecimiento $y$ diferenciación de las estructuras de la semilla. Estas condiciones o requerimientos son: humedad, temperatura $\mathrm{y} \mathrm{O}$, mismos que rompen con la quiescencia de la semilla o su estado de reposo. (Arellano y col., 2006).

Una de las formas de medir la eficiencia en la germinación, es la tasa de germinación de las semillas que se utilizan en una prueba con diferentes factores a evaluar, como por ejemplo el sustrato. Barrera y col., (2010) explican que el análisis de crecimiento es una aproximación cuantitativa, que usa datos simples y básicos, para la descripción e interpretación de las plantas que crecen bajo ambiente natural, seminatural o controlado.

\section{Materiales y métodos}

\section{Elaboración de composta}

Se utilizó un total de $80 \mathrm{~kg}$ de estiércol para cada composta y $40 \mathrm{~kg}$ de residuos vegetales por estiércol (borrego y conejo), también se utilizaron $20 \mathrm{~kg}$ de hojas de bambú por cada $40 \mathrm{~kg}$ de residuos vegetales frescos. Los materiales utilizados se mezclaron con pala, con la finalidad de obtener una consistencia homogénea y lograr el balance de partículas $\mathrm{C} / \mathrm{N}$ en la mezcla, se adicionaron $450 \mathrm{~g}$ de levadura (Saccharomyces cerevisiae Meyen ex E.C.Hansen) más $1 \mathrm{~kg}$ de azúcar estándar. Se utilizaron $20 \mathrm{~L}$ de agua para cada una de las mezclas de estiércol. 


\section{Material vegetal}

Se utilizaron semillas de albahaca (Ocimum basilicum) previamente colectadas en el vivero escuela y semillas de orégano silvestre (Lippia graveolens) colectadas en Colotlán, Estado de Jalisco

\section{Tratamientos}

Se realizaron cinco tratamientos, los cuales se describen en el Cuadro 1.
Posteriormente se establecieron las semillas de ambas especies en los tratamientos, utilizando 10 plantas por unidad experimental con tres repeticiones. Cada unidad experimental consistió en 10 plantas en una caja petri, que fueron dispuestas en estufa de germinación BINDER ${ }^{\circledR}$ sin temperatura controlada.

Cuadro 1. Tratamientos utilizados

\begin{tabular}{lccc}
\hline ESPECIE & \multicolumn{2}{l}{ TRATAMIENTOS } & \\
\hline \multirow{2}{*}{ Albahaca } & 1 & 2 & 3 \\
& Testigo & Jal $+15 \%$ borrego & Jal $+30 \%$ borrego \\
& & 4 & 5 \\
Orégano & 1 & Jal $+15 \%$ conejo & Jal $+30 \%$ conejo \\
& Testigo & Jal $+15 \%$ borrego & Jal $+30 \%$ borrego \\
& & 4 & 5 \\
& & Jal $+15 \%$ conejo & Jal $+30 \%$ conejo \\
\hline
\end{tabular}

\section{Diseño experimental y análisis de datos}

Se utilizó un diseño experimental completamente al azar, con cinco tratamientos $\mathrm{y}$ tres repeticiones por tratamiento. Los datos obtenidos fueron analizados utilizando el programa MINITAB ${ }^{\circledR}$, con el que se realizaron análisis de varianza y posteriormente se realizó la comparación múltiple de medias de los tratamientos por medio de la prueba de Tukey.

\section{Resultados y Discusión}

\section{Parámetros de germinación}

Los resultados obtenidos para la albahaca (Figura 1), son satisfactorios en los tratamientos testigo, y los tratamientos que contenían composta de borrego $30 \%$ y conejo 15\%. Según estos resultados, la aplicación de $15 \%$ de composta de borrego no es la causa de que disminuyera el porcentaje de germinación en esta especie, es probablemente un efecto aleatorio el que provocó que fuera el tratamiento con algunas semillas dañadas. 


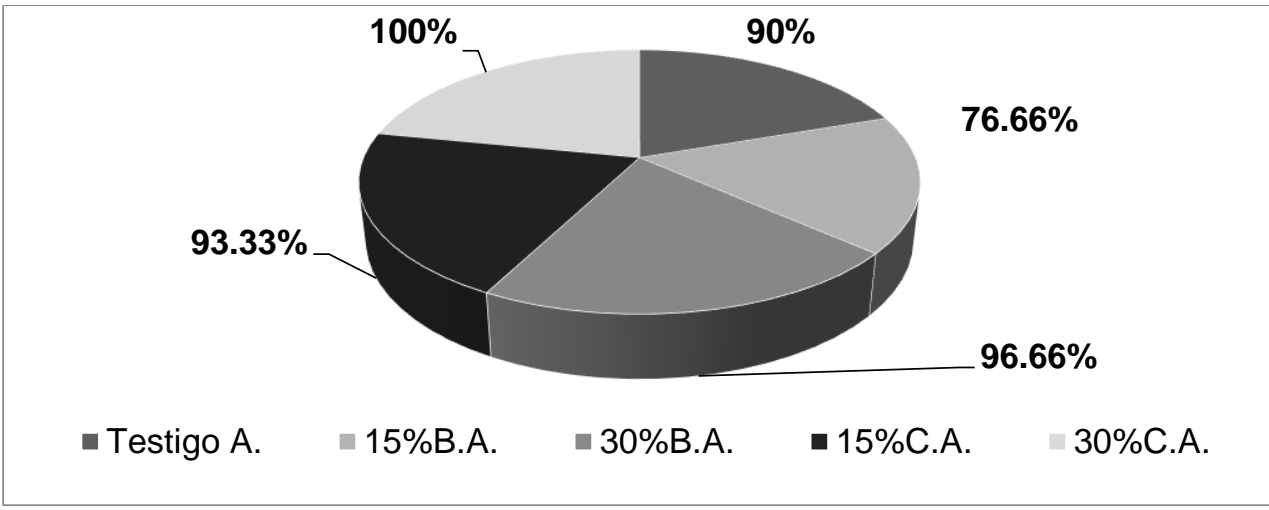

Figura 1. Porcentajes de germinación para cada tratamiento en albahaca.

Para el caso del orégano (Figura 2), se presentaron porcentajes de germinación muy bajos, cuyos valores fluctúan entre los 10 y $33.3 \%$, siendo el tratamiento $15 \%$ C.O. (con composta de conejo) el que presentó una influencia marcada sobre el número de semillas que germinaron.

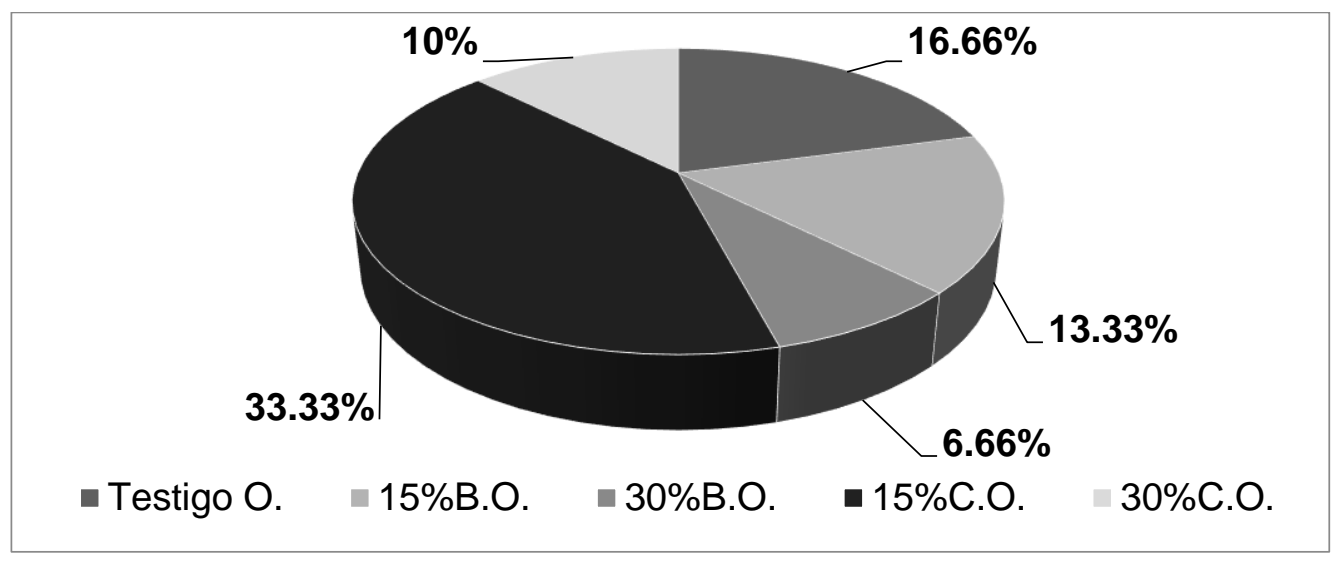

Figura 2. Porcentajes de germinación para cada tratamiento en orégano. 
En el Cuadro 2, se presenta un resumen de los resultados para las variables de germinación medida en el cual se observan en conjunto los efectos de los tratamientos en cada especie.

Cuadro 2. Comparación del efecto de los parámetros de germinación medidos en los tratamientos utilizados en el presente trabajo.

\begin{tabular}{lllll}
\hline Especie & Composta & $\begin{array}{l}\text { Porcentaje de } \\
\text { germinación }\end{array}$ & $\begin{array}{l}\text { Días a } \\
\text { germinación }\end{array}$ & $\begin{array}{l}\text { Tasa de } \\
\text { germinación }\end{array}$ \\
\hline Albahaca & Testigo & 90 & 2 & 0.0157 \\
& B. A.15\% & 76.6 & 2 & 0.0136 \\
& B. A. 30\% & 96.6 & 2 & 0.0197 \\
& C.A. 15\% & 93.3 & 2 & 0.0172 \\
Orégano & C.A. 30\% & 100 & 2 & 0.0364 \\
& Testigo & 16.6 & 3 & 0.1378 \\
& B.O. 15\% & 13.3 & 3 & 0.1066 \\
& B.O. 30\% & 6.6 & 4 & 0.0577 \\
& C.O. $15 \%$ & 33.3 & 3 & 0.0274 \\
& C.O. 30\% & 10 & 4 & 0.0337 \\
\hline
\end{tabular}

Análisis de varianza y pruebas de Tukey

Para cada uno de los parámetros medidos por especie, se realizó un análisis de varianza y posteriormente una prueba de Tukey con nivel de significancia 0.05 para discriminar las diferencias entre medias de cada tratamiento que resultó con diferencias significativas, según la metodología propuesta por Steel y Torrie (1985).

De los parámetros analizados, solamente se encontraron diferencias significativas en longitud del brote para albahaca (Cuadro 4) y para el caso de orégano, sólo la variable peso seco (Cuadro 8). Para albahaca, el tratamiento que tuvo incidencia directa en la longitud del brote, fue el testigo y para la variable peso seco de orégano, fue el tratamiento adicionado con $30 \%$ de composta de borrego.

Los resultados de los análisis de varianza, se encuentran en los Cuadros 3 al 12, de los cuales el Cuadro 5 y 9 son las pruebas de significancia de medias para las únicas variables que presentaron diferencia significativa. 
Cuadro 3. Resultados del análisis de varianza para peso seco $(\mathrm{mg})$ de albahaca

\begin{tabular}{llllll}
\hline & GL & SC & CM & F & P \\
\hline Trat & 4 & 3.0954 & 0.7738 & 1.42 & 0.290 n.s \\
Repetición & 2 & 1.3281 & 0.6641 & 1.22 & 0.332 \\
Error & 11 & 5.9765 & 0.5433 & & \\
Total & 17 & 10.4000 & & & \\
\hline
\end{tabular}

Cuadro 4. Resultados del análisis de varianza para longitud del brote de albahaca

\begin{tabular}{llllll}
\hline & GL & SC & CM & F & P \\
\hline Trat & 4 & 3.5081 & 0.8770 & 8.57 & $0.002^{* *}$ \\
Repetición & 2 & 0.0685 & 0.0343 & 0.33 & 0.723 \\
Error & 11 & 1.1262 & 0.1024 & & \\
\hline Total & $\mathbf{1 7}$ & $\mathbf{4 . 7 0 2 8}$ & & & \\
\hline
\end{tabular}

Cuadro 5. Prueba de Tukey con un $95,0 \%$ confianza para longitud de brote de albahaca.

\begin{tabular}{|c|c|c|c|}
\hline Trat & $\mathbf{N}$ & Lo & \\
\hline Testigo & 3 & 4.9 & A \\
\hline B.A. $\mathbf{3 0 \%}$ & 3 & 4.7 & A B \\
\hline B.A. $15 \%$ & 3 & 4.1 & $\begin{array}{l}\mathrm{A} \\
\mathrm{BC}\end{array}$ \\
\hline C.A. $15 \%$ & 3 & 4.0 & $\begin{array}{l}\mathrm{A} \\
\mathrm{BC}\end{array}$ \\
\hline C.А. $30 \%$ & 6 & 3.8 & $\mathrm{C}$ \\
\hline
\end{tabular}

Tratamientos que no comparten una letra son significativamente diferentes

Cuadro 6. Resultados del análisis de varianza para longitud de raíz de albahaca

\begin{tabular}{llllll}
\hline & GL & SC & CM & F & P \\
\hline Trat & 4 & 0.3916 & 0.0977 & 0.52 & 0.723 n.s \\
Repetición & 2 & 0.1118 & 0.0559 & 0.30 & 0.748 \\
Error & 11 & 2.0625 & 0.1875 & & \\
Total & 17 & 2.5649 & & & \\
\hline
\end{tabular}

Cuadro 7. Resultados del análisis de varianza para relación brote/raíz de albahaca

\begin{tabular}{llllll}
\hline & GL & SC & CM & F & P \\
\hline Trat & 4 & 6.036 & 1.509 & 1.07 & 0.418 n.s \\
Repetición & 2 & 3.481 & 1.741 & 1.23 & 0.329 \\
Error & 11 & 16.562 & 1.415 & & \\
\hline
\end{tabular}




\begin{tabular}{lll}
\hline Total & 17 & 25.080 \\
\hline
\end{tabular}

Cuadro 8. Resultados del análisis de varianza de peso seco en $(\mathrm{mg})$ de orégano

\begin{tabular}{llllll}
\hline & GL & SC & CM & F & P \\
\hline Trat & 4 & 0.6708 & 0.1677 & 3.74 & $0.023^{*}$ \\
Error & 17 & 0.7623 & 0.0448 & & \\
Total & 21 & 1.4332 & & & \\
\hline
\end{tabular}

Cuadro 9. Prueba de Tukey con un $95.0 \%$ confianza para peso seco $(\mathrm{mg})$ de orégano

\begin{tabular}{|c|c|c|c|}
\hline Trat & $\mathbf{N}$ & Peso s & \\
\hline Testigo & 6 & 0.6667 & B \\
\hline B.O. $15 \%$ & 10 & 0.7900 & B \\
\hline B.O. $30 \%$ & 1 & 1.5000 & A \\
\hline C.O. $15 \%$ & 3 & 0.7000 & B \\
\hline C.O. $30 \%$ & 2 & 0.6000 & B \\
\hline
\end{tabular}

Tratamientos que no comparten una letra son significativamente diferentes

Cuadro 10. Resultados del análisis de varianza para longitud del brote de orégano

\begin{tabular}{llllll}
\hline & GL & SC & CM & F & P \\
\hline Trat & 4 & 1.830 & 0.457 & 1.64 & 0.210 n.s \\
Error & 17 & 4.741 & 0.279 & & \\
Total & 21 & 6.571 & & & \\
\hline
\end{tabular}

Cuadro 11. Resultados del análisis de varianza para longitud de raíz de orégano

\begin{tabular}{llllll}
\hline & GL & SC & CM & F & P \\
\hline Trat & 4 & 0.434 & 0.108 & 0.99 & 0.438 n.s \\
Error & 17 & 1.856 & 0.109 & & \\
Total & 21 & 2.290 & & & \\
\hline
\end{tabular}

Cuadro 12. Resultados del análisis de varianza para la relación brote/raíz de orégano

\begin{tabular}{llllll}
\hline & GL & SC & CM & F & P \\
\hline Trat & 4 & 0.648 & 0.162 & 1.19 & 0.352 n.s \\
Error & 17 & 2.320 & 0.136 & & \\
Total & 21 & 2.969 & & & \\
\hline
\end{tabular}


Los resultados obtenidos, hacen evidente el efecto positivo de la composta de conejo en concentraciones de $30 \%$ para la albahaca y $15 \%$ para el orégano. Se observan resultados concluyentes sólo para una de las variables en cada caso y ambas están relacionadas con la acumulación de solutos osmóticos para provocar el incremento en peso seco y la elongación posterior a la extensibilidad de las paredes celulares (Azcón-Bieto y Talón, 2008; Barrera y col., 2010; Melgarejo, 2010).

\section{Conclusiones}

Con base en los resultados obtenidos, se encontró que es efectivo adicionar composta a base de estiércol de conejo para incrementar el porcentaje de germinación de semillas de Lippia graveolens. Lo anterior mejora la capacidad de germinación de la misma. Así mismo, se encontró que la adición de composta a base de estiércol de borrego, incrementa la producción de biomasa en las plántulas de orégano silvestre. Para albahaca, se recomienda la utilización de estiércol de conejo en una concentración de $30 \%$ en el sustrato para obtener porcentajes de germinación del orden del $100 \%$. Como perspectiva, se puede proponer el uso de composta para la germinación de semillas, así como aumentar los trabajos experimentales en otras especies aromáticas.

\section{Literatura citada}

Arellano. L., E. Sandoval, J. Sánchez, J.M. Padilla, M. Arriaga y A.N. Avedaño. (2006). Control de calidad de semillas. Manual. Universidad de Guadalajara. pp: 90.

Azcón-Bieto, J. y M. Talón. (1985). Fundamentos de fisiología vegetal. McGraw Hill. 2a . Ed. Madrid, España. pp: 651

Barrera, J. D. Suárez y L.M. Melgarejo. (2010). II. Análisis de crecimiento en plantas. En: Experimentos en fisiología vegetal. Universidad Nacional de Colombia. ISBN: 978958-719-668-9

Castellanos, O., G. Acevedo, M. Torres, F. Zurita, M. Gutiérrez, C. Del Toro y A. Rodríguez. (2013). In vitro clonal propagation and regeneration of the comercially important plant mexican oregano (Lippia graveolens). In Vitro Cell.Dev.Biol. 49: pp: 620-625.

De Luna, V.A. y E. Vázquez A. (2009). Elaboración de abonos orgánicos. Ed. Universidad de Guadalajara. pp: 86.

Melgarejo, L.M., (2010). Experimentos en fisiología vegetal. Universidad Nacional de Colombia. ISBN: 978958-719-668-9

Solano, M., F. Iriarte, P. Ciria y M.J. Negro. (2001). Performance characteristics of three aereation system in the composting of sheep manure and Straw. J. Agric. Engng. Res. 79(3): pp: 317-329.

Steel, R.G. y J. Torrie. (1985). Bioestadistica: principios y procedimientos. McGrawHill. pp: 620. 